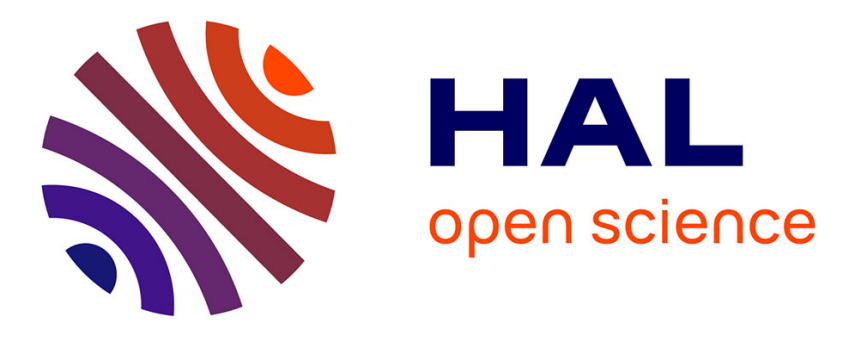

\title{
A Unifying Framework for Homogeneous Model Composition
}

Jörg Kienzle, Gunter Mussbacher, Benoit Combemale, Julien Deantoni

\section{To cite this version:}

Jörg Kienzle, Gunter Mussbacher, Benoit Combemale, Julien Deantoni. A Unifying Framework for Homogeneous Model Composition. Software and Systems Modeling, 2019, pp.1-19. 10.1007/s10270018-00707-8 . hal-01949050

\section{HAL Id: hal-01949050 https://hal.inria.fr/hal-01949050}

Submitted on 9 Dec 2018

HAL is a multi-disciplinary open access archive for the deposit and dissemination of scientific research documents, whether they are published or not. The documents may come from teaching and research institutions in France or abroad, or from public or private research centers.
L'archive ouverte pluridisciplinaire HAL, est destinée au dépôt et à la diffusion de documents scientifiques de niveau recherche, publiés ou non, émanant des établissements d'enseignement et de recherche français ou étrangers, des laboratoires publics ou privés. 


\title{
A Unifying Framework for Homogeneous Model Composition
}

\author{
Jörg Kienzle • Gunter Mussbacher . \\ Benoit Combemale • Julien Deantoni
}

Received: date / Accepted: date

\begin{abstract}
The growing use of models for separating concerns in complex systems has lead to a proliferation of model composition operators. These composition operators have traditionally been defined from scratch following various approaches differing in formality, level of detail, chosen paradigm, and styles. Due to the lack of proper foundations for defining model composition (concepts, abstractions, or frameworks), it is difficult to compare or reuse composition operators. In this paper, we stipulate the existence of a unifying framework that reduces all structural composition operators to structural merging, and all composition operators acting on discrete behaviors to event scheduling. We provide convincing evidence of this hypothesis by discussing how structural and behavioral homogeneous model composition operators (i.e., weavers) can be mapped onto this framework. Based on this discussion, we propose a conceptual model of the framework, and identify a set of research challenges, which, if addressed, lead to the realization of this framework to support rigorous and efficient engineering of model composition operators for homogeneous and eventually heterogeneous modeling languages.
\end{abstract}

Keywords model composition · symmetric merge $\cdot$ event scheduling · event structures $\cdot$ separation of concerns

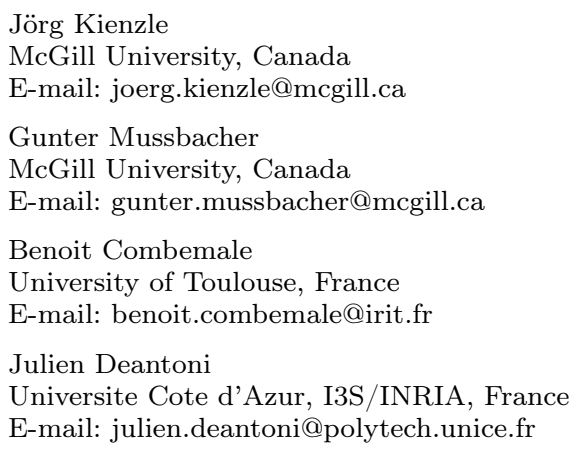




\section{Introduction}

Extending the time-honored practice of separation of concerns [14,39], ModelDriven Engineering (MDE) promotes the use of separate models to address the various concerns in the development of complex software-intensive systems [15]. The main objective is to chose the right level of abstraction to specify and reason about the system under development depending on stakeholder needs and system concerns. While some of these models can be defined with a single modeling language (e.g., UML), Domain-Specific Modeling Languages (DSMLs) are increasingly used to handle various concerns in system and software development [6]. To support this trend, the MDE community has developed advanced techniques for designing new DSMLs.

A consequence of separating concerns is that different, possibly heterogeneous, models need to be composed in order to execute the application or reason over global properties. In general, model composition unfolds along two dimensions, i.e, structure and behavior. So far, frameworks that offer composition operators had to define their own composition rules and provide custom-made implementations of their operators (e.g., through transformations).

Depending on the context of use, different or customized composition operators are needed to provide support for different development paradigms (e.g., incremental development or Software Product Line (SPL) development) and address the various objectives (e.g., analysis, compilation, runtime management, etc.) of the developer. Furthermore, due to the increasing number of application domains of interest, and the growing number of stakeholders, new DSMLs are constantly developed and new composition operators need to be developed accordingly. While dedicated foundations have been proposed in the last decade to systematically engineer modeling languages and more specifically DSMLs, this is not yet the case for defining the corresponding composition operators. Those foundations need to be elaborated in order to move from tedious, adhoc crafting of composition operators to structured, stream-lined engineering.

This paper proposes a framework for engineering composition operators that is based on the hypothesis that all structural composition can be expressed with symmetric merging, and all (discrete-event) behavioral composition can be reduced to asymmetric event scheduling. The framework introduces a canonical categorization of homogeneous model composition operators (i.e., weavers) and establishes a foundational set of capabilities required for most, if not all, DSMLs to support composition and modularization. Concretely, the contributions of this paper are (i) a clear definition of model composition via a clear definition of merging and event scheduling, (ii) a survey of existing approaches dedicated to homogeneous model composition w.r.t. this categorization, and (iii) a set of research challenges indicating how to realize the proposed model composition framework and outlining future research directions.

The structure of this paper is as follows. Section 2 illustrates the need for different homogeneous and heterogeneous composition operators by outlining examples in which structural and behavioral model composition is used in different contexts and for different purposes. Section 3 positions the proposed model composition framework in the landscape of composition techniques and describes four generic steps for composition operators. Section 4 then goes into further details of structural composition, while Section 5 discusses behavioral composition. Sec- 
tion 6 provides a description of the key concepts of the proposed model composition framework as part of the presentation of the set of research challenges. General purpose weavers are contrasted as related work with the proposed framework in Section 7. Finally, Section 8 concludes the paper.

\section{Illustrating Examples}

This section presents concrete real-world examples in which models and model composition have been used to separate concerns during development. Each situation required the definition and implementation of composition operators, either structural or behavioral, tailored to the application context of the composition and the notation(s) that had to be composed.

\subsection{Need for Different Homogeneous Composition Operators for Standard Modeling Notations}

Standard modeling notations, e.g., the Unified Modeling Language, have been used extensively in the context of MDE over the last two decades. While the UML specification document published by OMG [37] standardizes the notation, it does not specify in what context or for what purpose the notation should be used during software development. For example, class diagrams have been used in very different contexts that imply different composition strategies, e.g., for specifying objectoriented design structure, for specifying domain models, and even for defining the abstract syntax for modeling languages in form of meta-models.

While modeling and model-driven engineering continue to gain popularity, it soon also became clear that despite the power of abstraction of modeling, models of real-world problems and systems quickly grow to such an extent that managing the complexity by using proper modularization techniques becomes necessary [1]. As a result, many standard modeling notations have been extended with aspectoriented mechanisms to support advanced separation of concerns. Not surprisingly, for a given notation, depending on the purpose for which models are being used, different composition operators have been proposed in the literature.

A concrete example of this, adapted from [21], is illustrated in Figure 1. The top of the figure depicts two state diagrams, SD1 and SD2. The bottom left shows the result of composing $S D 1$ with $S D 2$ using the state diagram composition operator defined by the HiLA appoach [44]. The bottom right shows the result of composing the same state diagrams using the state diagram composition operator defined as part of the Protocol Modeling approach (PM) [30], which is in turn inspired by the CSP parallel composition operator $\|$.

The results of the composition are clearly different. HiLA is an approach where state diagrams are used in the context of low-level software design to describe the behavior of system components and generate code. In HiLA, states with matching names are merged, and transitions and states that only appear in one of the input models are copied. PM on the other hand is used during high-level requirements specification and analysis for simulation and test generation purpose. In this context, to ensure the tractability of protocol analysis and enable local reasoning, the || composition operator was designed to preserve the trace behavior of the input 


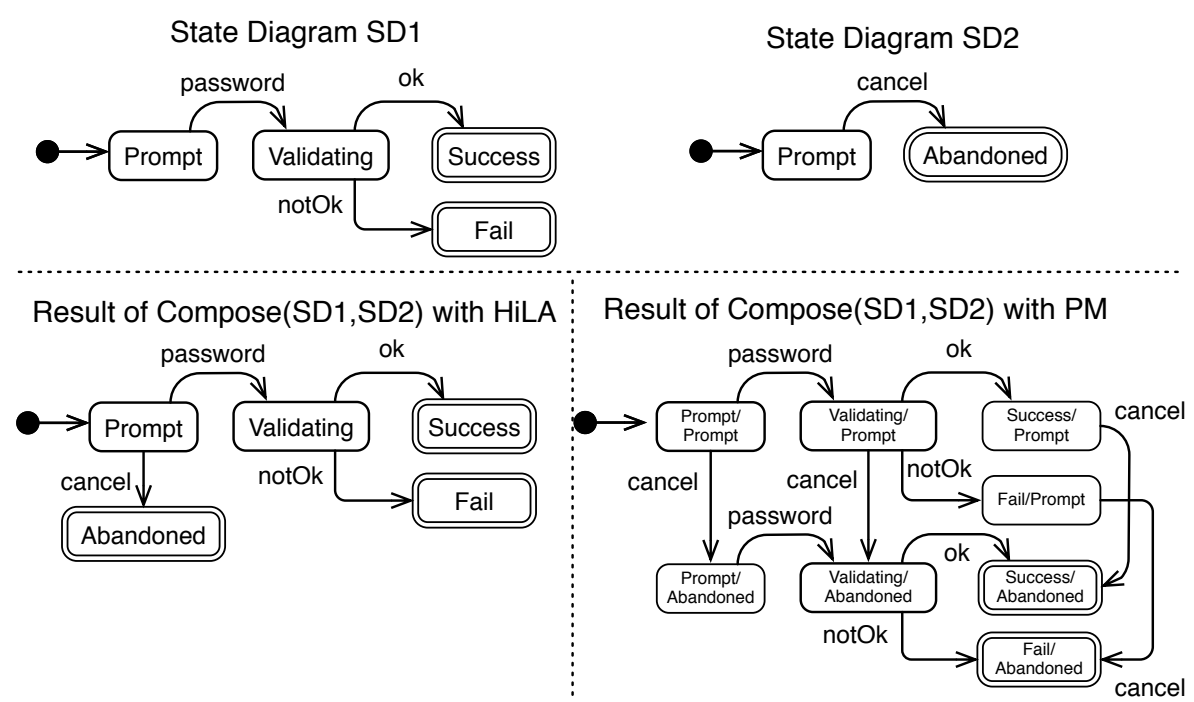

Fig. 1 Different Composition Operators for State Diagrams

models. In other words, composing another protocol with a given protocol $S D$ cannot override a constraint that $S D$ says must be true. The $\|$ composition operator ignores state names, and composes transitions that are equivalents in both input models by ensuring they are taken synchronously. In our specific example, since there are no equivalent transitions between SD1 and SD2, the composition result contains the Cartesian-product of the states in the two input models $\left(4^{*} 2\right.$ $=8$ states).

\subsection{Need for Heterogeneous Composition Operators between DSMLs}

The development of modern complex software-intensive systems often involves the use of multiple DSMLs that capture different system aspects. In addition, models of the system aspects are seldomly manipulated independently of each other. System engineers are thus faced with the difficult task of relating information presented in different models. For example, a system engineer may need to analyze a system property that requires information scattered in models expressed in different DSMLs. Current DSML development workbenches provide good support for developing independent DSMLs, but provide little or no support for integrated use of multiple composed DSMLs. The lack of support to explicitly relate concepts expressed in different DSMLs makes it very difficult for developers to reason about information spread across different models in one composed model.

Modern complex software-intensive systems increasingly use software as an integration layer. As a consequence, architectures require system-level models to integrate various engineering-specific architectures. For example, block diagrams describing device characteristics may have to be composed with class diagrams describing software systems.

Modern systems like cyber-physical systems and the Internet of Things are highly connected to the environment. Various DSMLs have been defined for de- 
scribing the system behavior on the one hand, and the environment or the physical world on the other hand. For example, state machines may be used to describe the behavior of a system (e.g., ThingML ${ }^{1}$ for embedded and distributed systems) and may have to be composed with sequence diagrams for interactions with the environment.

\section{The Landscape of Composition Operators}

The term composition is used in many situations [33]. At its most abstract, composition refers to the act of creating new entities from existing ones (e.g., by assembling together two or several smaller entities). This may occur at low levels of granularity (e.g., by adding an association or generalization between two classes) or at high levels of granularity (e.g., by connecting required and provided interfaces of components). In this paper, we are interested in composition in the context of MDE and DSMLs, i.e., in the composition of models at high levels of granularity. In other words, we are not interested in the composition of individual modeling elements such as a single class or state with another class or state, respectively, but at the composition of structural and behavioral models that represent broader concerns of interest to stakeholders. For space reasons, the paper further narrows the detailed discussions to the composition of homogeneous models (i.e., models defined by the same metamodel). While the initial focus is on homogeneous models, the intent is to investigate the applicability of our envisioned model composition framework to heterogeneous models in greater detail in the future.

Recently, aspect-oriented techniques have enabled advanced separation of concerns, i.e., they provide a developer with systematic means for the identification, separation, representation, and most importantly composition of crosscutting concerns. Aspect-oriented language extensions and dedicated composition operators (also known as weavers) have been defined for many programming languages and modeling languages. We evaluate our proposed model composition framework by mapping well-known model weavers (and hence a rich set of varied but clearly scoped composition operators) to the framework.

Many model composition operators have been proposed in the literature over the last two decades. In [29], Marchand et al. argue that any composition process can be reduced to four steps:

1. Optional pre-processing of the inputs,

2. Determining the composition location (either through implicit or explicit matching, and by intention or by extension),

3. Combining the inputs at the location(s) determined in step 2 to produce the output, and

4. Optional post-processing of the output.

It is therefore not surprising that existing composition operators define algorithms for executing these steps, where Step 1 and Step 4 are optional. This applies to structural compositions such as UML Package Merge [38], Kompose [16], RAM Class Diagrams [23], Theme/UML Class Diagrams [7], and AoGRL [34] as well as behavioral compositions such as ADORE [31], TreMer+ [35], RAM Sequence

\footnotetext{
1 Cf. http://thingml.org/
} 


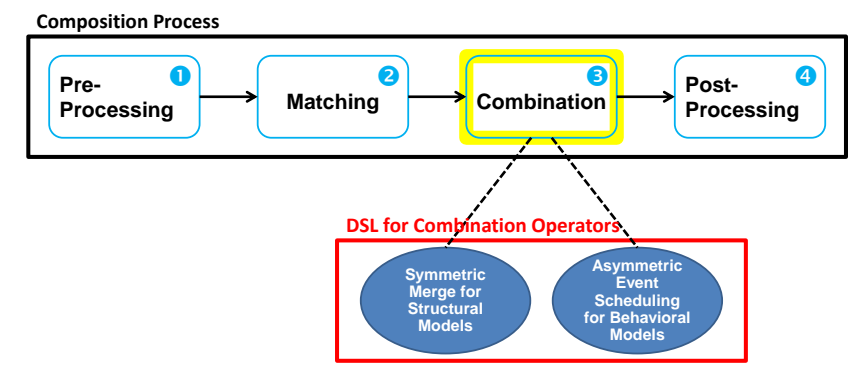

Fig. 2 Overview of Unifying Framework for Homogeneous Model Composition

Diagrams [23], Theme/UML Sequence Diagrams [7], HiLa [44], AoUCM [34], and RAM Protocol Models [2].

The pre-processing step (Step 1) may, e.g., rename model elements as in Kompose [16]. The matching step (Step 2) may involve the evaluation of a pattern as in AoGRL and AoUCM [34] (i.e., an implicit approach) or the establishing of an explicit binding as in RAM [23]. Furthermore, matches may be defined by intention (i.e., expressed at the language level) as in TreMer + [35] or by extension (i.e., expressed at the model level) as in Theme/UML [7]. Many other techniques have been discussed in literature that perform different kinds of matching with varying degrees of sophistication and could be applied in Step 2. The final post-processing step (Step 4) may involve applying transformation rules to address conflicting model elements as in UML Package Merge [38].

The following two sections on structural and behavioral combination focus on Steps 2 and 3, since they are mandatory steps that all operators need to provide and because this allows us to consistently identify commonalities and differences between the operators. All aspect-oriented modeling techniques mentioned explicitly in this section are presented in more detail in the following two sections (some in greater detail than others for readability reasons). We outline how in each case, the third combination step can always be mapped to a symmetric merge operation for structural models and asymmetric event scheduling for behavioral models. The aim is to provide convincing evidence that the hypothesis on which the vision of our framework is built is indeed true.

Figure 2 illustrates the proposed framework, including the composition process and highlighting the step of the process to which symmetric merge (discussed in Section 4) and asymmetric event scheduling (discussed in Section 5) apply as well as the early DSL for the definition of reusable composition operators (as proposed in Section 6).

\section{Structural Combination - Merge}

This section precisely defines the structural merge operator of our framework, and then shows in detail how it can successfully be applied to compose class diagrams. Then, further examples of how merge can provide composition for other modeling notations are outlined and the mathematical properties of merge are discussed. 


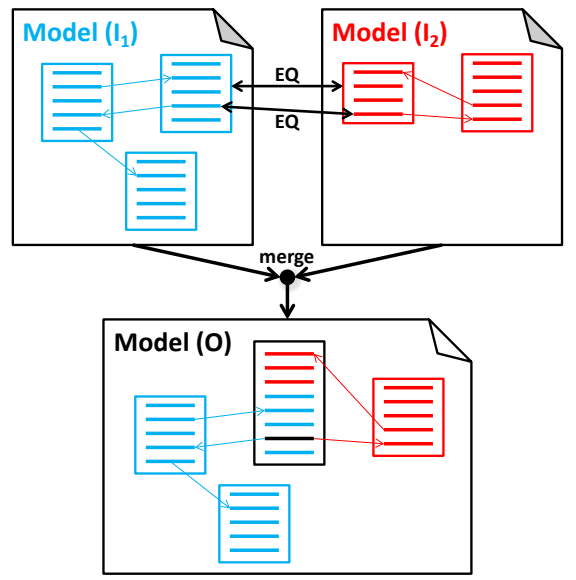

Fig. $3 \operatorname{merge}\left(I_{1}, I_{2}, E Q\right) \Rightarrow O$

\subsection{Definition}

Essentially, a structural model consists of elements (some of which are containers) with a set of properties, including relationships to other elements. The structural composition examples listed in Section 3 suggest that the combination operation for structural elements is always a symmetric merge. Formally, the merge combination operator takes two models $I_{1}$ and $I_{2}$ as inputs, as well as a set of equivalence relationships $E Q=e_{1} \Leftrightarrow e_{2}$ with $e_{1} \in I_{1}$ and $e_{2} \in I_{2}$. Each model element $e$ of input model $I$ has a finite set of properties $\left\{p_{e}\right\}$, which can refer to other model elements inside the same input model $I$. The merge combination operator produces a new output model $O$ that contains for each relationship between $e_{1}$ and $e_{2}$ in $E Q$ a single model element that has as a set of properties the union of the properties of the related elements, i.e., $\left\{p_{e 1}\right\}$ and $\left\{p_{e 2}\right\}$. All model elements in $I_{1}$ and $I_{2}$ that are not mentioned in $E Q$ are simply copied over into $O$. See Figure 3 for an illustration of symmetric merge.

\subsection{Examples of Structural Weavers}

Class Diagram Weaving: The Reusable Aspect Models approach (RAM) [23] defines two composition operators for composing class diagrams. In RAM, the software designer that elaborates a class diagram can incorporate structural elements defined in another class diagram by either reusing or extending the other class diagram. In the case of reuse, the reused class diagram exposes a set of model elements (classes, operations, parameters) that provide structural properties that are intended to be combined with model elements from the reusing class diagram in a so-called customization interface. The designer needs to map each element from the customization interface to the desired model element in the reusing model. In the case of extension, the extending class diagram can add additional properties to any model element of the extended class diagram, as well as define additional 


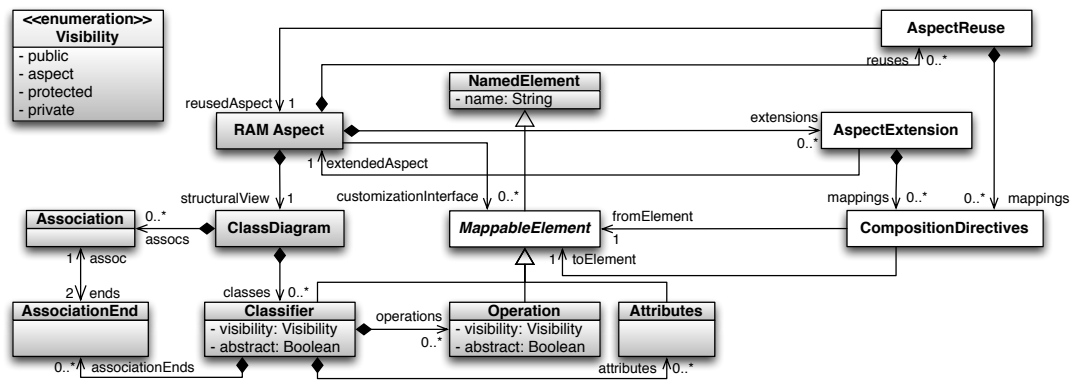

Fig. 4 Parts of the Class Diagram Metamodel of the RAM Modelling Approach

model elements. Here, any model elements with the same signature (e.g., name for classes, name and parameter types for operations, etc.) are combined by default, but the designer can specify additional mappings between elements of the extending model and the extended model, if desired.

A simplified metamodel of RAM Class Diagrams is shown in Figure 4 (classes highlighted in gray). RAM Aspect is the root of the containment hierarchy, and it contains one ClassDiagram, which is composed of many Classifiers, which in turn contain Operations and Attributes. For illustration purpose, the visibility and abstract properties for Classifier and Operation are shown. Other information, e.g., type information, parameters, etc., is omitted for readability reasons. A class diagram can also contain Associations, which are linked to two AssociationEnds that are contained in the Classifier that they belong to.

The classes in white are the ones that are used to specify composition. AspectReuse is used to specify a reuse composition, whereas AspectExtension is used to specify an extends composition. In both cases, the mappings (instances of CompositionDirective) specify which model element from the reused or extended aspect is mapped to which element in the current aspect, i.e., fromElement refers to a model element in the reused/extended class diagram, and toElement points to a model element in the current class diagram.

Figure 5 shows an example class diagram that models the structure of the Observer design pattern [17]. The Bank class diagram reuses the Observer by specifying a reuse composition specification that maps Subject $\rightarrow$ Account, modify $\rightarrow$ withdraw/deposit, Observer $\rightarrow$ AccountWindow and update $\rightarrow$ refreshWindow.

To produce the composed model, the RAM weaver creates a copy of the Bank aspect, and then deep-copies or merges the model elements of the Observer aspect into this new model according to the CompositionDirectives. In other words, it performs the merge operation described in subsection 4.1, where $I_{1}=$ Observer, $I_{2}$ $=$ Bank, and $E Q=$ instances of CompositionDirectives (see mappings in Figure 4).

In the case of reuse composition, the preprocessing step of the composition operator (see step 1 in section 3) changes the visibilities of the elements in the reused class diagram from public to package. The motivation for this is the information hiding principle [39]: the interface of the reusing class diagram should not expose structural elements of the reused class diagram. There is no need for the matching step, since the composition directives enumerate all elements that 


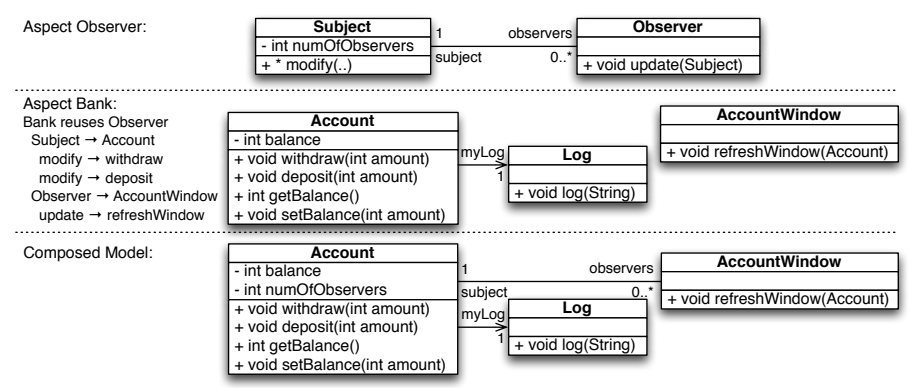

Fig. 5 Example Composition of Class Diagrams in the RAM Modelling Approach

are to be combined with our proposed merge operator. There is also no need for postprocessing the merged output.

In the case of extends composition, the matching step compares the model elements in the two input models to identify element pairs with the same signature. For each pair, a composition directive that maps one to the other is created and added to the AspectExtension. The augmented mappings are then passed to the RAM weaver, who as a result merges the model elements with identical signatures according to the semantics of RAM model extension.

UML Package Merge: Package merge has been introduced in UML2 to improve modularity [38] and is extensively used in the UML specification itself. The package merge composition operator takes as input two class diagrams, and extends the first with the second by merging their common classes, and deep copying the other ones. These common classes are identified by their names and types. The merge is done recursively, following the containment links of the models. The output of the merge replaces the first class diagram, hence the merge is asymmetric, in the sense that only the first model is modified. However, except for the place where the merged model is stored and for conflict resolutions applied during the post-processing step, the technique is symmetric. In other words, the combination step does not depend on the order of the inputs, and maps nicely to symmetric merge in the proposed framework in a similar way as explained above for RAM class diagrams.

Kompose: Kompose is a model composition tool [16] implemented in the Kermeta language [22]. It merges two homogeneous models by comparing the signatures of their elements. These signatures can be arbitrarily complex, using the element's name, type, field, or method names and types, and so on. Elements with the same signature are merged, while all other ones are deep copied. Kompose proposes a system of pre- and post-directives to modify the models before and after the merge. The two models are called base model and aspect model, which suggests an asymmetric treatment, yet the tool is symmetric [16]. The composition operator follows the four steps described in Section 3, and in particular, includes the symmetric merge combination operator.

Theme/UML: Theme/UML [7] for class diagrams is similar to the aforementioned techniques in that structural model elements are merged symmetrically with each other, in this case based on specified binding relationships for template parameters. The properties of bound elements are merged, while non-template-parameter 
elements are added to the composed result. The approach of Theme/UML matches the proposed framework in its use of a symmetric merge combination operator.

AoGRL: The Aspect-oriented and Goal-oriented Requirement Language (AoGRL) [34] matches a parameterized goal model fragment (i.e., a pattern) against the base goal model to identify locations where aspectual goal model elements are to be inserted into the base model. While the specification of the composition is asymmetric, the actual operator is symmetric once the insertion locations have been identified, because conceptually pattern elements from the aspect are merged with matched elements from the base. AoGRL therefore adheres to the proposed framework.

\subsection{Mathematical Properties of Merge}

The merge combination operator inherits its mathematical properties from the union operator on which it is based. It is therefore commutative (merge $(a, b)=$ $\operatorname{merge}(b, a))$ and associative $(\operatorname{merge}(a, \operatorname{merge}(b, c))=\operatorname{merge}(\operatorname{merge}(a, b), c))$.

Nevertheless, the composition operators of the different approaches listed above are not necessarily commutative, i.e., they are not necessarily symmetric. This stems from the fact that the preprocessing, matching, and postprocessing steps of many composition operators perform operations that are not commutative.

For example, in RAM, the reuse composition operator changes the visibilities of the elements in the reused class diagram from public to package in the preprocessing step. The motivation for this is the information hiding principle [39]: the interface of the reusing class diagram should not expose structural elements of the reused class diagram. This pre-processing step implies $R A M$ reuse $(a, b) \neq$ $R A M$ reuse $(b, a)$, because the visibility of the model elements in the composed model would be different.

Conflict resolution also sometimes results in asymmetry, although different approaches handle conflict resolution in different ways. This is due to the fact that what constitutes a conflict depends highly on the modelling language. Conflicts can be resolved during preprocessing or postprocessing (like it is done in Kompose or UML Package Merge, where conflicting elements must be renamed before the symmetric merge happens), but also by choosing different models that are to be composed (as it is done in RAM).

Finally, some composition operators perform in-place update, i.e., they modify one of the input models during composition, which renders them asymmetric. For example, UML package merge composes one model into the other one, or in other words, the composed model replaces one of the inputs. This is orthogonal to the merge operation proposed in our framework. In our framework, after the models are composed, the user of the framework can decide whether to store the resulting model separately, or whether to overwrite one of the input models.

Some AOM approaches, e.g. MATA [42], allow an aspect to also remove elements from the base model. Since our structural composition is based on merge, it supports additive composition only. Removing elements is not of compositional nature, and related to program or model slicing [4]. Slicing is a technique used, for example, to extract parts of a system so that it can be reused in a different context, or when using negative variability in software product line development. 
To support removing of elements, our framework could be combined with a generic slicer such as [4], but this is out of the scope of this paper.

\section{Behavioral Combination - Event Scheduling}

This section defines event structures and the behavioral event scheduling operator of our framework, and then shows in detail how it can successfully be applied to compose sequence diagrams, state diagrams, and aspect-oriented use case maps. Then, further examples of how event scheduling can provide composition for other modeling notations are outlined.

\subsection{Definition}

A behavioral model is a model where its structure (elements and properties) represents behavior, possibly non-deterministic or concurrent. Such behavior can be obtained by applying the operational semantics of the language used to build the behavioral model on the model (i.e., by executing the model). Such process is for instance detailed in [26] or [10].

The behavioral composition examples listed in Section 3 act on behavioral input models and create a new behavioral model that specifies a particular interleaving of the input models' behaviors. Therefore, even if the composition operators take as input the behavioral models, they actually need to infer an internal representation of the execution of the input models to reason about their interleaving. Independently, this internal representation can be inferred statically (with dedicated analysis) or dynamically (simulation).

To reason on concurrent behaviors, concurrency theory introduced causal (also called true-concurrent) representations. A causal representation captures the concurrency, dependency and alternative relations among actions in a particular behavior. A well known instance of such representation is Event Structure [36,43]. An event structure represents a partial order of events specifying the causality relations as well as alternative relations between actions of concurrent behavior. This fundamental model totally abstracts data and model structure to concentrate on the partial ordering of its actions. This model is not expressive enough to encode (continuous) timed or synchronous behaviors and there exist several extensions of this model, e.g., tagged signals [27] or the time model [3]. In this paper, however, we choose to present the framework based on event structures since it is expressive enough for the examples that follow and allows for simpler explanations. In any case, all the explanations and definitions given in the remainder of the paper are also appropriate for more powerful fundamental models.

Due to its nature, an event structure is a means to apply abstraction to any model by concentrating only on the observable actions in the model. An event structure is independent of the abstraction level since an event can abstract any kind of action (from the entering into a state to the call of an arbitrarily complex action). Due to this independence from the abstraction level, the same model can be represented by different, more or less detailed, event structures. For the same reason, there are usually different models that can be represented in an abstract way by the same event structure. 


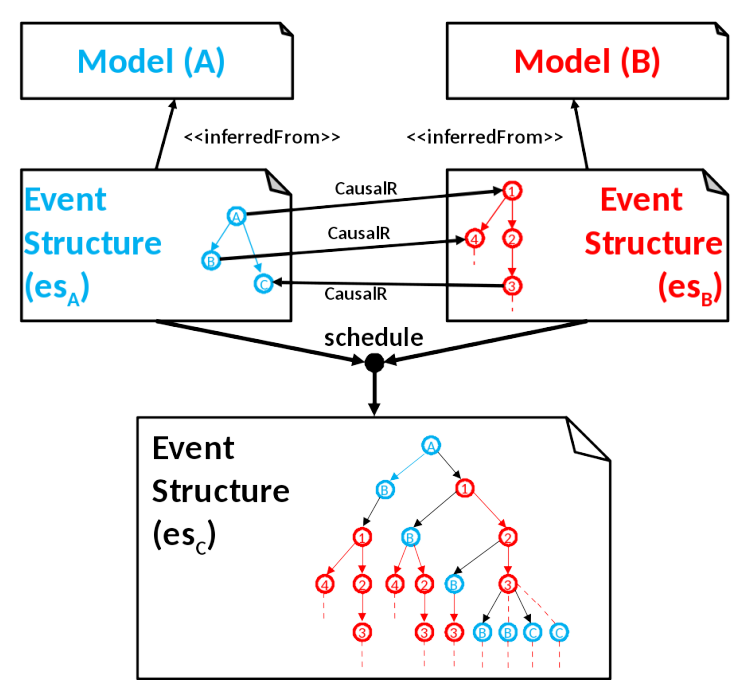

Fig. 6 schedule $\left(e s_{A}, e s_{B}\right.$, CausalR $) \Rightarrow e s_{C}$

We believe that the combination operation for two behavioral models $A$ and $B$ can always be reduced to event scheduling that operates on the two event structures describing the behavior of $A$ and $B$. Formally, the event scheduling operator takes as input two event structures $e s_{A}$ and $e s_{B}$ representing the behavior of models $A$ and $B$, as well as a set of causal relationships between events of different models Causal $R:=e_{j} \rightarrow e_{k}$. If $e_{j}$ is an event from $e s_{A}$, then $e_{k}$ must be an event from $e s_{B}$, or vice versa. $e_{j} \rightarrow e_{k}$ means that $e_{k}$ must take place at the same time or after $e_{j}$. The result of applying the schedule combination operator is a combined event structure $e s_{C}$ that takes the event relationships CausalR into account. See Figure 6 for an illustration of event scheduling, where the events and dependencies of $e s_{A}$ are shown in blue, the events and dependencies of $e s_{B}$ in red, and the causal relationships between events from $e s_{A}$ and $e s_{B}$ are shown with black arrows.

Before continuing, the reader should note that an event structure specifies all possible concurrent or alternative execution paths of the model. In an event structure, two alternative execution paths at a specific point in time (i.e., from a specific configuration of the event structure) are usually represented by two causalities to different event occurrences, creating two different paths that do not contain any causality to a common event occurrence (even if from some point they have the same future, see middle of Figure 7). On the other hand, two concurrent execution paths at a specific point in time are represented by two causalities to different event occurrences, creating two different paths that will eventually be causality related to a common event occurrence in the event structure (see left of Figure 7).

To represent alternative execution paths in a more compact way than duplicating all common futures, we use a dummy node marked 'A' in an event structure. The outgoing causalities of the dummy node denote exclusive execution paths, even if they are eventually causally related to a common event occurrence. The use of the dummy node is illustrated on the right side of Figure 7 . The figure on the left describes a situation where after event $a$ two event sequences $(b . . x)$ and 


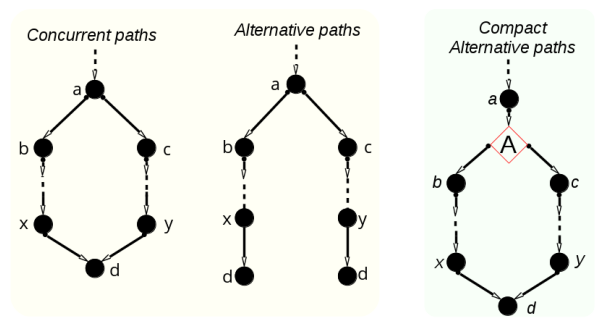

Fig. 7 Usual and Compact Representation of Alternatives

(c..y) occur potentially in parallel, and then synchronize again for $d$. On the other hand, the event structure shown in the middle represents a situation where two possible futures exist after the occurrence of $a$, one starting with $b$ and the other one starting with $c$. In the case where both futures have a common event sequence at some point in the future, the dummy node allows us to join the two branches at the first common event ( $d$ in our case as illustrated by the model on the right) instead of having to show two distinct sub-trees.

\subsection{Examples of Behavioral Weavers}

In this section we illustrate the eventscheduling operator on three different examples. These examples have been chosen to evaluate the applicability of our approach on different kinds of behavioral models (inspired by the UML behavioral models). The first one is Sequence Diagram, a message based behavioral language, the second one is State Diagram, a state based behavioral language and the third one is AoUCM, a workflow based behavioral language (as UML activity diagrams). We end the section by briefly explaining how the proposed framework can be applied on existing approaches that are proposing a composition operator.

Sequence Diagram Composition: RAM allows a software designer to express behavior using sequence diagrams [23]. In sequence diagrams, instances of objects, represented by lifelines, send messages to each other. The important behavioral events are the message send and message receive events. For each lifeline, a causal ordering is specified for the message reception and message sending events. Between lifelines, another causal ordering specifies that a receive event of a message cannot occur before the corresponding send event has occurred. While sequence diagrams can be used to depict both asynchronous and synchronous sending of messages, they are always used in RAM to specify the interactions in form of synchronous operation invocations that happen between objects as a result of an operation call.

To support advanced separation of concerns, RAM allows the modeler to specify object interactions pertaining to different concerns in separate sequence diagrams, and use composition to generate the combined behavior when needed. For instance, if the behavior of operations $p$ and $q$ is described in separate sequence diagrams, and if somewhere within the model of $p$ the operation $q$ is invoked, then the RAM sequence diagram weaver can combine the two models to produce a new model that depicts the combined flow of execution by "inlining" the communication of $q$ within $p$ at the right place. 


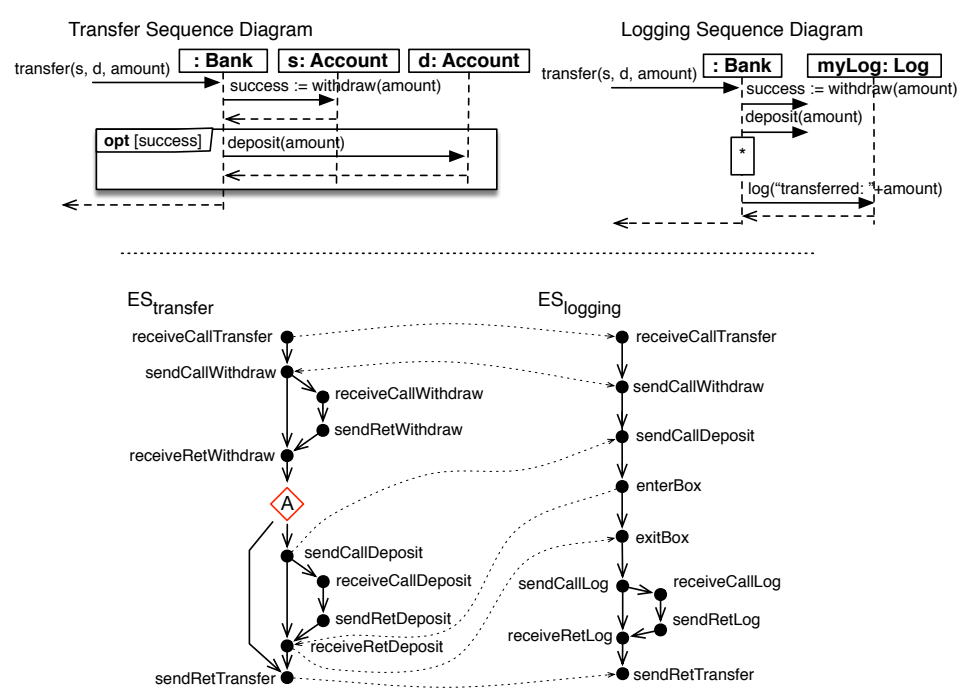

Fig. 8 RAM Sequence Diagram Composition

RAM also supports aspect-oriented composition of sequence diagrams, an example of which is shown in Figure 8. On the top left of the figure, a simple RAM sequence diagram is shown that specifies the behavior of the transfer operation of the Account class previously shown in Figure 5. It simply calls the withdraw operation of Account $s$, and, in case the withdraw was successful then calls deposit on Account t. The corresponding event structure ES $S_{\text {transfer }}$ is depicted underneath the sequence diagram. The first event on the : Account lifeline is the reception event of the transfer call, which is followed by the send event of the withdraw call, followed by the reception event of the return of the withdraw call as well as the receive event of the call of withdraw, etc.

A separate sequence diagram, shown on the top right of Figure 8, depicts the behavior of Logging. It specifies that whenever transfer is invoked, followed by a call to withdraw, followed by a call to deposit, then the remaining behavior of transfer is first executed (depicted by a rectangular box containing a $\star$ ). After that, the successful transfer is logged by calling log provided by myLog. The event structure $E S_{\text {logging }}$ corresponding to the Logging sequence diagram is shown underneath. Note that the rectangular box with the $\star$ was converted into two events.

To compose the two behaviors, additional causal relationships are added between the events of ES transfer and ES logging. They are shown as dotted arrows in Figure 8. For all events that match in both event structures a causal dependency from the base model to the aspect model is created, i.e., $\forall e_{i} \in$ $E S_{\text {transfer }}, e_{j} \in E S_{\text {logging }} \mid$ match $\left(e_{i}, e_{j}\right) \Longrightarrow\left(e_{i} \rightarrow e_{j}\right) \in$ CausalR. In this example, this is true for the events receiveCallTransfer, sendCallWithdraw, sendCallDeposit and sendRetTransfer. Since the location of the rectangular box with the $\star$ in the Logging sequence diagram determines when the execution of the remaining behavior of transfer should occur, two additional causal relationships have to be added to CausalR: from the enterBox aspect model towards the first remaining event occurring on the Bank lifeline in the transfer model 


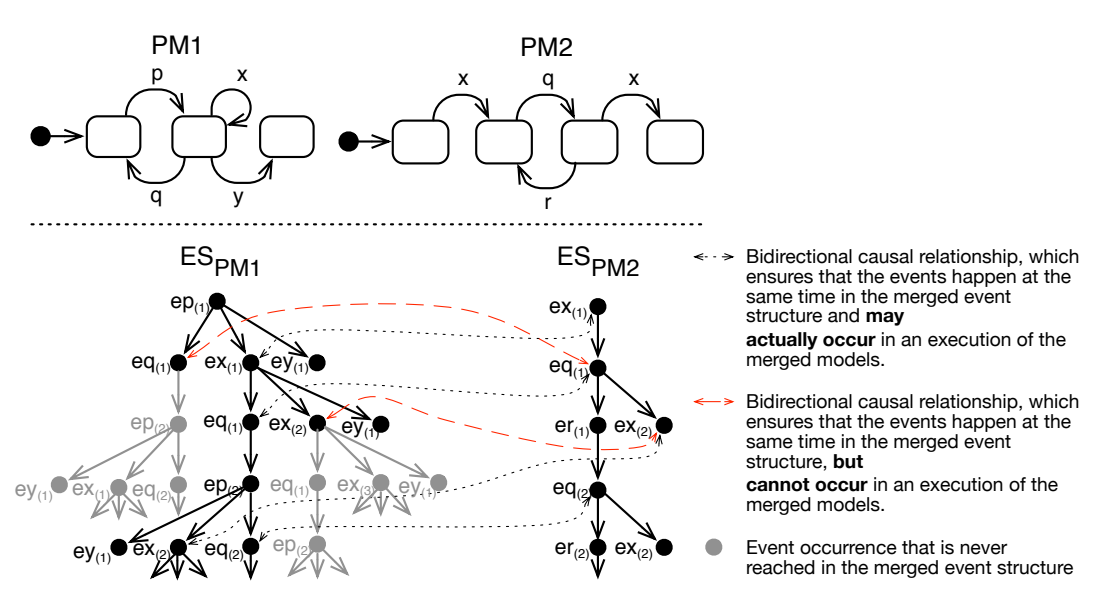

Fig. 9 RAM State Diagram Composition

(enterBox $\rightarrow$ receiveRetDeposit), as well as from the last event of the execution of transfer in the base model towards the exitBox event in the aspect model (receiveRetDeposit $\rightarrow$ exitBox).

This simple example illustrates also that our behavioural weaving framework can support semantic weaving. In case the withdraw operation is unsuccessful, the sendCallDeposit event never occurs, and hence the behaviour of the logging aspect is never executed.

State Diagram Composition: RAM allows a software designer to specify operation invocation protocols for each design class using state views [2], which are a variant of protocol state diagrams called protocol models [30]. A state view comprises a set of states and a set of named transitions that stand for the operations that the class declares. For instance, a state view comprising two states for a File class might specify that a file initially starts in a "Closed" state, waiting for a call to the open operation, which transitions to the "Opened" state. There, the operations read and write are available, until the close operation is called, which brings the file back to the "Closed" state. In protocol modeling terms, the "Closed" state accepts open and rejects read, write, and close, whereas the "Opened" state does the inverse. Operations that are not mentioned in a state view are ignored, i.e., no decision on whether to accept or reject them is taken.

A state view is mapped to an event structure by creating an event et for every transition $t$ in the model. The event structure is then given by the tree containing all possible event occurrence scenarios acceptable by the state view starting from the initial state. For state views with cycles the resulting event structure is usually infinite.

Figure 9 illustrates the transformation from state view to event structure on an example. In the state view $P M 1$, the first transition that can occur is $p$. This is why the event structure $E S_{P M 1}$ has the event $e p_{(1)}$ at the $\operatorname{root}^{2}$. After $p$, transition $q, x$, or $y$ can be taken in the state view. $E S_{P M 1}$ therefore contains $e q_{(1)}, e x_{(1)}$ and $e y_{(1)}$, and $e p_{(1)} \rightarrow e q_{(1)}, e p_{(1)} \rightarrow e x_{(1)}$ and $e p_{(1)} \rightarrow e y_{(1)}$. If transition $q$ is

\footnotetext{
2 We are using the subscript ${ }_{(i)}$ to emphasize that the dots in the event structure represent event occurrences and not event types.
} 
taken, then $p$ can be taken again. Therefore, $E S_{P M 1}$ contains another occurrence of transition $p$, namely the event occurrence $e p_{(2)}$ and $e q_{(1)} \rightarrow e p_{(2)}$. The resulting infinite tree represents all possible executions of the state view.

When the structural weaver of RAM merges two classes, resulting in the union of the operations of the classes as explained in Section 4.2, the RAM protocol weaver also needs to combine the two state views associated with the classes to yield the state view that specifies how to correctly use the new merged class.

The rules for combining the two state views is equivalent to the CSP parallel composition operator typically denoted $\|$. It specifies that for an operation to be accepted by $I_{1} \| I_{2}$, both $I_{1}$ and $I_{2}$ must either accept or ignore the operation. In other words, if either $I_{1}$ or $I_{2}$ rejects the operation, then $I_{1} \| I_{2}$ also rejects the operation.

In RAM, to combine two event structures representing two state views, it suffices to add causal relationships among the event occurrences of the sets of transitions $T_{P M 1}$ and $T_{P M 2}$ that match. More precisely, $\forall t 1 \in T_{P M 1}, \forall t 2 \in$ $T_{P M 2} \mid \operatorname{match}(t 1, t 2) \Longrightarrow\left(\forall e t 1_{i} \in E S_{P M 1}\right.$, et $2_{i} \in E S_{P M 2},\left(\left(\right.\right.$ et $1_{i} \rightarrow$ et $\left.2_{i}\right) \in$ CausalR $\wedge\left(\right.$ et $2_{i} \rightarrow$ et $\left.1_{i}\right) \in$ CausalR $)$ with et $_{i}$ defined as the ith event occurrence of the transition $t$ ). The composition of the state views $P M 1$ and $P M 2$ is shown at the bottom of Figure 9 with the additional arrows representing some of the introduced causal relationships between the event structures $E S_{P M 1}$ and $E S_{P M 2}$. Whenever there are causal dependencies in both directions, it is ensured that they have to happen simultaneously.

Aspect-oriented Use Case Map Composition: Aspect-oriented Use Case Maps (AoUCM) [34] is a scenario/workflow notation that employs a pattern-based approach to identify locations in the base model where aspectual behavior is to be inserted. Since AoUCM models capture causal relationships, they are straightforwardly transformed into event structures by denoting each AoUCM model element $m$ as an event em (except for those AoUCM model elements that represent purely control flow information and are hence directly mapped onto causal relations instead of events).

The AoUCM approach features an enhanced matching algorithm that takes semantic equivalences in the UCM notation into account and allows interleaving of scenarios. However, once the insertion location has been determined by the sophisticated matching algorithm, the actual composition of the aspectual and base behavior is always accomplished by the insertion of an aspect marker, which acts as a reference in the base model to the aspectual behavior that needs to be inserted.

The example AoUCM model [32] was selected, because it features a more complex composition based on interleaved scenarios. The base AoUCM model on the left of Figure 10 shows the scenario for ordering a video online, comprised of the steps selectMovie, processOrder, payForMovie, and sendMovie. The aspectual model in the middle defines the movie points scenario, which is interleaved with the base behavior with the help of the diamond shaped pointcut stubs. Each pointcut stub represents a pattern that is matched against the base model, e.g., the processOrder pointcut stub is matched against the processOrder step in the base model (the actual definition of the pattern is not shown in the figure). The movie points scenario requires a membership form to be filled out before processing an order, allows movie points to be redeemed instead of paying for the movie, and 
enables earning of movie points after sending a movie if the transaction was not a redemption.

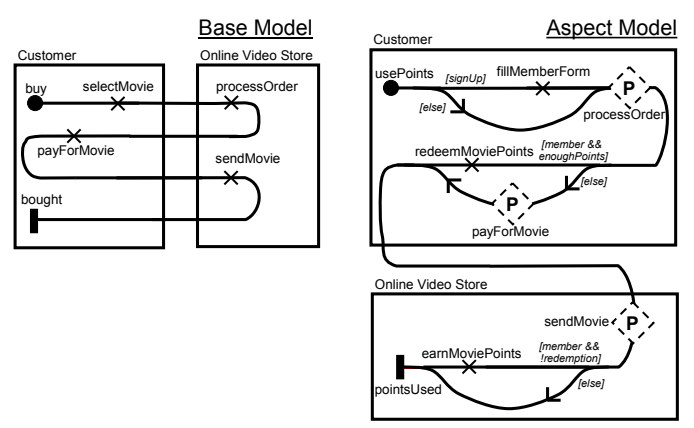

\section{Composed Model}

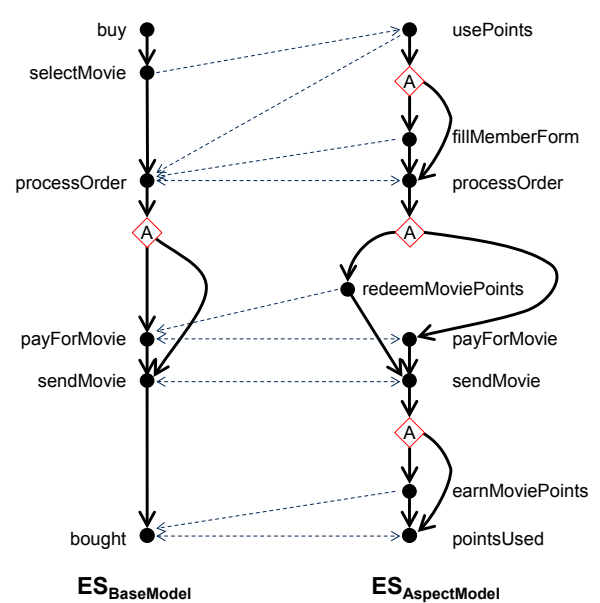

Fig. 10 AoUCM Scenario/Workflow Composition

At the bottom of Figure 10, the combined event structures of the AoUCM base and aspect model are shown. The event structure of the base model is represented on the left side, the one of the aspect model on the right side, and the causalities required by the combination of both models are shown in the middle. First of all, each pattern match is synchronized, e.g., the processOrder event from the base model is synchronized with the processOrder event from the aspect model. This is equivalent to the synchronization of events performed for RAM sequence diagrams. Then, additional causal relationships are expressed in the aspectual event structure for segments created by the synchronized elements. Three types of segments exist. A segment may (a) start at a start point and end at a synchronized element (e.g., from usePoints to processOrder), (b) start and end at a synchronized element (e.g., from processOrder to sendMovie), or (c) start at a synchronized element and end at an end point (e.g., from sendMovie to pointsUsed). 
The first element in a segment has an additional causal relation from a base model element (i.e., usePoints) or is a synchronized element (i.e., processOrder, sendMovie). Additional causal relations never have to be specified for already synchronized elements. If the first element in a segment is not a synchronized element, then the causal relation exists from the base model element immediately preceeding the base model element synchronized with the aspectual element at the end of the segment (i.e., selectMovie $\rightarrow$ usePoints).

If the segment ends with a synchronized element, then all elements in the segment have a causal relation to the base element synchronized with the end of the segment (e.g., usePoints $\rightarrow$ ProcessOrder, fillMemberForm $\rightarrow$ ProcessOrder, and redeem MoviePoints $\rightarrow$ sendMovie). However, if the segment ends in an end point, then all elements have a causal relation to the base element immediately following the base model element synchronized with the aspectual element at the start of the segment (e.g., earnMoviePoints $\rightarrow$ bought, pointsUsed $\rightarrow$ bought).

AoUCM allows base behavior to be replaced by aspectual behavior (e.g., redeemMoviePoints may replace payForMovie). Therefore, the event structure of the base model needs to be augmented with alternatives to allow base elements to be skipped (see the 'A' node in the event structure of the base model).

More precisely, during the translation from a model to its event structure, the expressiveness of the weaver must be taken into account and made explicit in the resulting event structure. Because the AoUCM weaver allows for removing (i.e., skipping) some actions from the original model, the translation of any AoUCM model must contain, between each event occurrence, an alternative allowing to skip one or more event occurrences. This does not only apply to AoUCM but to any weaver of a behavioral model that allows replacement (e.g., it is also necessary for RAM sequence diagrams to support around advice). While these additional skip causalities may appear as a limitation of our proposed framework, we believe, on the contrary, that the skip causalities make explicit something often hidden deep inside the core of a weaver and which is now made visible to any tool in a generic way (e.g., to a generic analysis tool). Skip causalities could for instance be used by a model checker to conclude that the base model respects a property, but that a specific aspect could lead to a property violation. Even more interesting, considering property violations, the concerned alternative could be removed, effectively reducing the expressiveness of the weaver to ensure that no aspect can violate a specific property. In the composed model in Figure 10, all possible skip causalities are not shown to simplify the representation. The shown skip causality (i.e., the 'A' node) in the event structure of the base model corresponds to the one from the aspect model that resides in the same segment, and is the only one that is relevant for the composed model in this example.

ADORE: ADORE is a tool for service orchestration using PROLOG. It allows the scheduling of partial orchestrations (fragments) in a main orchestration using a set of user-defined relationships [31]. A fragment essentially represents partially ordered service calls and ends, which can be represented through an event structure. The relationships between fragments which describe the orchestration correspond to the asymmetric combination operation eventscheduling of our framework, which acts thanks to event relationships between the event structures inferred from the input fragments.

TreMer+: In [35], Nejati et. al. propose an approach, implemented in TreMer+, to merge statechart diagrams while preserving their semantics by ensuring bisim- 
ulation. Thus, the operator is not only structural, but also ensures a particular relationship between the behaviors that represent the statechart diagrams. A statechart diagram is representing a partial order of events, naturally represented by an event structure. Concurrent actions, e.g., regions in statechart diagrams, can be represented by concurrent branches in the event structure, and synchronizations between concurrent machines can be ensured by causalities in the event structure Then, the combination operation infers the required event relationships (CausalR) between the two input event structures to ensure a bissimilar combined event structure (based on a powerful matching step).

Theme/UML: Theme/UML [7] for sequence diagrams is very similar to RAM in that the combined flow of execution for two sequence diagrams also involves proper "inlining". Therefore, the behavioral composition can be expressed the same way as for RAM.

HiLa: The High-Level Aspects for UML State Machines (HiLA) approach [44] targets the modeling of use case scenarios with UML state machines, enhanced with aspect-oriented modeling features. To derive the corresponding event structure, inputs coming from the environment are mapped to events. Synchronization between events in the base model and the aspect model can be achieved with two inverse causal relationships. HiLa also provides what is called dynamic aspects that can compose state machines based on the execution trace. For instance, a so-called «history» property allows a modeler to specify a constraint that checks, for instance, that a certain state was entered more than $n$ times in order to trigger behavior expressed in the aspect state machine. To achieve this composition using event structures, a causal relationship enabling the first event of the aspect state machine must be scheduled in all event orders of the base machine that visit the state more than $n$ times.

\section{Discussion}

\subsection{Conceptual Model}

In Sections 4 and 5, we gave evidence of foundational primitives for structural and behavioral model combination that can handle many composition operators developed for a variety of modeling languages. In Fig. 11, we summarize these foundations as an early DSL that could be used for defining reusable model composition operators in the future.

A Combination Operator can be either a Structural combination operator between Model Elements of Structural Models, or a Behavioral combination operator between Events of the Combination Event Structure. The combination event structure possibly extends the Event structure inferred from the execution of (i.e., specifiedBy) Behavioralmodels, with additional Combination Events that represent a combination of events for a particular purpose of the combination operator.

A structural combination operator is composed of Equivalence Mappings between two unordered model elements, while a behavioral combination operator is composed of CausalRelationship between a source event and a target event. Of course this event relationship can be completed by more specific ones (e.g., mutual exclusion, temporal relationships). 


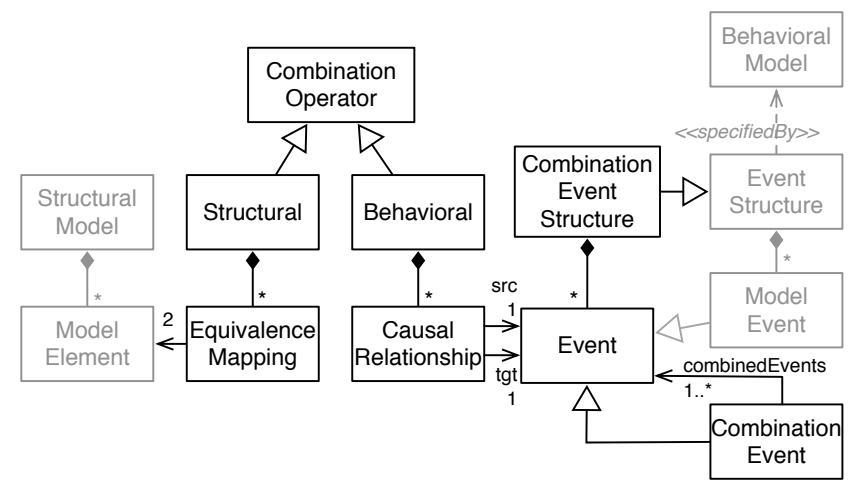

Fig. 11 Foundational Primitives for Model Composition

The vision we have proposed in this paper takes the form of a complete framework to support rigorous and efficient engineering of homogeneous and potentially heterogeneous model composition operators. If successful, the proposed framework would provide a reusable building block for (modeling) language design efforts, and streamline the creation and application of domain-specific languages with support for separation of concerns in the context of MDE.

\subsection{Research Challenges}

As outlined in section 3, the execution of composition operators can be decomposed into 4 steps: preprocessing, matching, combining, and postprocessing. The discussion of this paper has focused mostly on the most important step of this process, step 3, which addresses the actual combination of models. We argued that it can be provided in a generic way by a symmetric merge operator for structural models and an asymmetric event scheduling operator for behavioral models after they have been transformed into event structures. In order to realize the entire proposed vision, several research challenges remain:

- C1: Ensure that all behavior models (that we want to be able to compose in the context of MDE) can be mapped to corresponding event structures.

- C2: Establish proof that our hypothesis (structural combination = merge, behavioral combination $=$ event scheduling) is correct.

- C3: Render all steps of the composition operators as generic / reusable as possible.

- C4: Provide efficient automation where possible.

- C5: Operationalize the proposed composition operator framework by integrating it with a language engineering workbench.

\subsection{Proposed Work Items}

The challenges to which the following six work items contribute are shown in parentheses in the header of each individual work item. 
W1: Mapping to Event Structures (C1)

We already conducted experiments that represent other behavioral models with specific kind of events structures $[12,13]$. To contribute further to $\mathrm{C} 1$, additional behavioral modeling languages should be mapped onto event structures.

W2: Automate Mapping to Event Structures (C4)

To contribute to $\mathrm{C} 4$, the application of the mapping from individual models to event structures needs to be automated by defining and implementing concrete model transformations. In this context, we have already published algorithms that create a symbolic event structure that encodes all the possible event structures of a behavioral model in $[19,20,28]$. Finally, to avoid constructing the symbolic event structure manually, we used a specification of the language execution semantics suitable to generate the symbolic event structure of any model conforming to the language $[9,11]$.

\section{W3: Implementing Composition Operators (C2)}

With initial tool support in place, additional evidence to support $\mathrm{C} 2$ needs to be provided by implementing the homogeneous composition operators surveyed in this paper according to the thoughts outlined in sections 4 and 5 with our framework. For heterogeneous composition, we have previously experimented with the coordinated execution of event structures in [18]. Vara Larson et al. [40] uses a symbolic representation of event structures to conjointly execute different models according to a specific coordination. Most recently, we also proposed the coordination of heterogeneous models based on rules expressed at the language semantics-level in [41].

\section{W4: Towards Generic Behavioral Matching (C3)}

While for all the behavioral model composition operators surveyed in this paper the matching step is notation-specific, we hypothesize that it does not need to be. In fact, instead of transforming a behavioral model into an event structure after the matching (step 2) and before combining (step 3), the transformation could be performed after the notation-specific preprocessing (step 1). This would allow exploring the possibility of defining a generic behavioral matcher that operates solely on event structures. Intuitively, both common input options for matching mechanisms - explicit bindings or patterns - can typically be translated into event structures following mapping rules similar than the ones used to map the input models to event structures themselves. In this case, a generic event pattern matcher would be able to identify any locations of interest in the behavior, provided that all potential matching points of the notation (i.e., the join points in aspect-oriented terms) are represented in the form of events in the event structure. Since event structures represent the execution of behavior, an additional advantage of matching at this level is that patterns that occur in the execution can be detected (i.e., the semantic interpretation of a model), as opposed to detecting patterns in the model itself (i.e., the abstract syntax of the model). For instance, such a matcher would be very convenient to match patterns that are known to require loop unrolling in the context of sequence diagram weaving as described in [24] or an understanding of the semantic equivalences of hierarchical decomposition as described in [34].

Providing generic matching capabilities in the context of our proposed framework would significantly contribute to $\mathrm{C} 3$.

W5: Efficient Creation of the Combined Event Structure (C4, C5)

To further contribute to $\mathrm{C} 4$ and to the operationalization of the framework as 
stated for C5, combined event structures need to be created efficiently. In the case where the event structure obtained from a behavioral model is finite, e.g., for Figures 8 and 10, the combined event structure is also finite and can easily be created offline (i.e., not at runtime). A first work item concerns the creation of the combined event structure for behavioral models with infinite event structure (e.g., the one of Figure 9). In this case, it is not possible to create the infinite event structure offline and consequently not possible to create the combined event structure "statically". However, it is possible to provide a framework to coordinate the execution of the base and the aspect model executions. It is then necessary to monitor the execution of each model and to setup a runtime matcher that triggers the creation of EventRelationship elements at runtime. The simulation framework must then be able to implement such new relations on the fly. Using a runtime matcher can be costly in time so that a second work item concerns the finite encoding of infinite event structure. While this has been done earlier in $[19,20,28]$, their encoding was very low-level so that it is not well adapted for composition reasoning. Our current idea is to investigate a simple 'folding' node (a kind of parametrized jump) allowing finite representation of infinite event structure, while supporting generic composition. The final goal is to propose a generic, static model weaver supporting finite and infinite event structures with or without loop unrolling and other semantic-based compositions.

\section{W6: Visualizing the Composed Behavior (C5)}

In addition, to be fully equivalent to existing behavioral composition operators, it does not always suffice to create a composed event structure that exhibits the correct composed behavior. To contribute to the operationalization of the framework as stated for C5, it is often important to be able to visualize the composed behavior in the original modeling formalism. The visualization takes place after the four steps discussed in this paper. A bi-directional mapping from a modeling language to event structures and back would allow the causal relations between the base and the aspect model to be indicated with tags or markers. An examination of the causal relations between the base and aspect model identifies the locations in the event structure, where aspectual behavior is added. This can then be translated into a location in the original modeling formalism with the help of the bi-directional mappings. The composed model could then be indicated by showing a visual element either before, at, or after the location in the original modeling formalism.

Consider, e.g., the case of AoUCM's weaver [32,34], which results in the placement of aspect markers in the base model as shown in Figure 12. The locations where aspect markers need to be inserted in the base model are identified by the synchronized events in the event structures of the base model and their bidirectional mappings back to the source base model. The type of aspect marker that needs to be inserted depends on the type of aforementioned segments in the event structure of the aspect model. A segment with a start point or end point results in an aspect marker with an outgoing link to the aspect model and an incoming link from the aspect model (see, e.g., aspect marker A and F in Figure 12). For segments where both the start and the end are synchronized elements, aspect markers with only an outgoing link (e.g., B and D) or only an incoming link (e.g., $\mathrm{C}$ and $\mathrm{E}$ ) are inserted into the base model depending on whether the aspect marker corresponds to the start or end of the segment, respectively. 


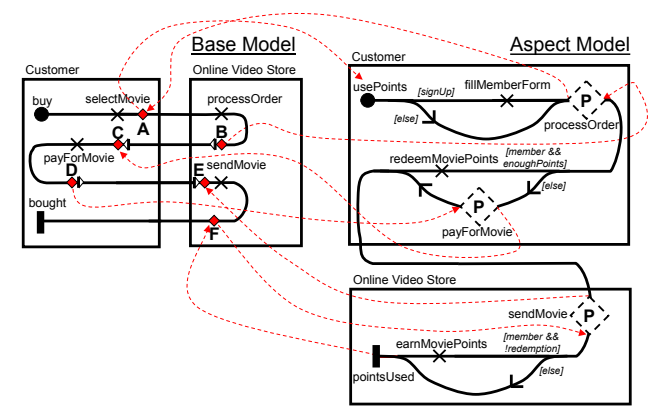

Composed Withdraw+Logging Behavior:

Fig. 12 Visualization of Composed Model with Tags/Markers (AoUCM) or Inline (RAM Sequence Diagrams)

Similarly, a weaver for RAM sequence diagrams [23] that is based on their event structures would identify the location of inserted behavior by the location of the causal relations to enterBox and exitBox and the existence of aspectual behavior specified before or after these two relations, e.g., after in the case of the sequence diagram in Figure 8. Consequently, behavior needs to be inserted into the identified base model location based on the bi-directional mappings from the event structure of the aspectual model to the source model. For notations such as sequence diagrams that can be layouted automatically, it is then even possible to show the inserted behavior inline in the base model as shown in Figure 12.

\section{Related Work: General Purpose Weavers}

This section discusses the shortcomings of existing tools and frameworks which may be used to combine two models with each other.

GeKo: GeKo is a generic, extensible model weaver that can compose any models that conform to a common metamodel [25]. It takes as parameters a base model, a pointcut model (which can specify a pattern), and an advice model, and replaces all instances of the pointcut model that are found in the base model with the advice model. The mappings between base and advice are inferred by the weaver by comparing model element properties. The combination of models that is done for each pointcut match is equivalent to our merge, i.e., it operates on the structure of the model only. As a result, GeKo, although applicable to behavioral models, cannot perform semantic-based combination.

MATA: MATA is similar to GeKo in that it can compose any models conforming to a common metamodel, but uses graph transformations to do so [42]. Similarly to GeKo, MATA operates on model structure and cannot perform semantic-based combination, which is possible with event structures.

ModMap: ModMap is a mapping language to express bi-directional translation between models conforming to object-oriented metamodels [8]. The mapping language allows for creating relationships between any metamodel element such as classes, attributes, or relations between classes. The interpretation mechanism is 
achieved through the assignment of a strategy to a given mapping. Strategies in the ModMap language are operations which allow the alignment of the two models involved. These operations are either predefined (rename an element, concatenate one or several elements with strings, add or remove an element from a collection) or defined by the user with a provided action language. While ModMap offers one generic merge algorithm, there is no consideration of behavioral semantics.

Event-Based Modularization: In [5], the authors present how an aspect-oriented approach can be augmented with an explicit specification of events as a way to express pointcuts. They show how such pointcut events can be combined to express conditions on the weaving of an advice. The authors propose few generic operators between events, but mainly focus on high-level operators for decomposition.

In contrast to existing general purpose weavers, our composition framework addresses structural models as well as the semantics of behavioral models, which is crucial as complex systems always have to be defined and evolve along both structural and behavioral dimensions.

\section{Conclusion}

The growing use of domain-specific languages and the need to combine instances of such languages leads to a continued demand for customized composition operators. This paper discusses a unifying framework that reduces all structural composition operators to structural merging, and all composition operators acting on discrete behaviors to event scheduling. The framework aims to support the definition and reuse of composition operators and avoid having to define them from scratch for each use. Based on a discussion of the properties of structural and behavioral composition operators and an analysis of how existing model composition operators can be mapped onto our proposed framework, we introduce a conceptual model of the framework and enumerate a set of research challenges that need to be addressed to realize the proposed framework and support rigorous and efficient engineering of model composition operators for homogeneous and eventually heterogeneous modeling languages. In future work, we will address the work items related to the five research challenges discussed in Section 6. Furthermore, we will investigate how heterogeneous composition operators can be supported by our proposed framework.

\section{References}

1. Aspect-Oriented Modeling Workshop Series. http://www.aspect-modeling.org/.

2. Wisam Al Abed, Matthias Schöttle, Abir Ayed, and Jörg Kienzle. Concern-oriented behaviour modelling with sequence diagrams and protocol models. In Behavior Modeling Foundations and Applications, volume 6368 of LNCS. Springer, 2015.

3. Charles André, Frédéric Mallet, and Robert De Simone. Modeling time(s). In Proceedings of the 10th International Conference on Model Driven Engineering Languages and Systems, MODELS'07, pages 559-573, Berlin, Heidelberg, 2007. Springer-Verlag.

4. Arnaud Blouin, Benoit Combemale, Benoit Baudry, and Olivier Beaudoux. Kompren: Modeling and Generating Model Slicers. Software and Systems Modeling, 14(1):321-337, 2015 
5. Christoph Bockisch, Somayeh Malakuti, Mehmet Akşit, and Shmuel Katz. Making aspects natural: Events and composition. In 10th International Conference on Aspect-oriented Software Development (AOSD '11). ACM, 2011.

6. Christopher Bull and Jon Whittle. Supporting reflective practice in software engineering education through a studio-based approach. IEEE Software, 31(4):44-50, 2014.

7. Siobhán Clarke and Robert J. Walker. Generic aspect-oriented design with Theme/UML. In Aspect-Oriented Software Development, pages 425-458. Addison-Wesley, 2005.

8. Mickael Clavreul, Olivier Barais, and Jean-Marc Jézéquel. Integrating legacy systems with MDE. In 32nd ACM/IEEE International Conference on Software Engineering (ICSE'10), pages 69-78. ACM, 2010.

9. Benoit Combemale, Julien Deantoni, Matias Vara Larsen, Frédéric Mallet, Olivier Barais, Benoit Baudry, and Robert France. Reifying Concurrency for Executable Metamodeling. In Martin Erwig, Richard F. Paige, and Eric Van Wyk, editors, SLE - 6th International Conference on Software Language Engineering, volume 8225, pages 365-384, Indianapolis, IN, United States, October 2013. Springer.

10. Julien Deantoni, Papa Issa Diallo, Ciprian Teodorov, Joël Champeau, and Benoit Combemale. Towards a Meta-Language for the Concurrency Concern in DSLs. In Design, Automation and Test in Europe Conference and Exhibition (DATE), Grenoble, France, March 2015

11. Julien Deantoni, Papa Issa Diallo, Ciprian Teodorov, Joël Champeau, and Benoit Combemale. Towards a Meta-Language for the Concurrency Concern in DSLs. In Design, Automation and Test in Europe Conference and Exhibition (DATE), Grenoble, France, March 2015.

12. Julien Deantoni and Frédéric Mallet. TimeSquare: Treat your Models with Logical Time. In Sebastian Nanz Carlo A. Furia, editor, TOOLS - 50th International Conference on Objects, Models, Components, Patterns - 2012, volume 7304, pages 34-41, Prague, Czech Republic, May 2012. Czech Technical University in Prague, in co-operation with ETH Zurich, Springer.

13. Julien Deantoni, Frédéric Mallet, Frédéric Thomas, Gonzague Reydet, Jean-Philippe Babau, Chokri Mraidha, Ludovic Gauthier, Laurent Rioux, and Nicolas Sordon. RT-simex: retro-analysis of execution traces. In Kevin J. Sullivan Gruia-Catalin Roman, editor, SIGSOFT FSE, volume ISBN 978-1-60558-791-2 of Proceedings of the 18th ACM SIGSOFT International Symposium on Foundations of Software Engineering, pages 377-378, Santa Fe, United States, November 2010.

14. Edsger Wybe Dijkstra. A discipline of programming, volume 1. Prentice-Hall Englewood Cliffs, 1976.

15. Douglas C. Schmidt. Model-Driven Engineering. IEEE Computer, 39:41-47, 2006.

16. R. France, F. Fleurey, R. Reddy, B. Baudry, and S. Ghosh. Providing support for model composition in metamodels. In EDOC, pages 253-264, 2007.

17. Erich Gamma, Richard Helm, Ralph Johnson, and John Vlissides. Design Patterns. Addison Wesley, Reading, MA, USA, 1995.

18. Kelly Garcés, Julien Deantoni, and Frédéric Mallet. A Model-Based Approach for Reconciliation of Polychronous Execution Traces. In SEAA 2011 - 37th EUROMICRO Conference on Software Engineering and Advanced Applications, Oulu, Finland, August 2011. IEEE.

19. Calin Glitia, Julien Deantoni, and Frédéric Mallet. Logical Time @ Work: Capturing Data Dependencies and Platform Constraints. In Tom J. J. Kaźmierski and Adam Morawiec, editors, System Specification and Design Languages, volume 106 of Lecture Notes in Electrical Engineering, pages 223-238. Springer New York, 2012.

20. Arda Goknil, Julien Deantoni, Marie-Agnès Peraldi-Frati, and Frédéric Mallet. Tool Support for the Analysis of TADL2 Timing Constraints using TimeSquare. In ICECCS'2013 18th International Conference on Engineering of Complex Computer Systems, Singapore, Singapore, July 2013.

21. Matthias Hölzl, Alexander Knapp, and Gefei Zhang. Modeling the Car Crash Crisis Management System Using HiLA, pages 234-271. Springer Berlin Heidelberg, Berlin, Heidelberg, 2010.

22. Jean-Marc Jézéquel, Benoit Combemale, Olivier Barais, Martin Monperrus, and FranÃ ğois Fouquet. Mashup of metalanguages and its implementation in the Kermeta language workbench. Software \& Systems Modeling, 2013.

23. Jörg Kienzle, Wisam Al Abed, and Jacques Klein. Aspect-Oriented Multi-View Modeling. In 8th International Conference on Aspect-Oriented Software Development (AOSD'O9), pages 87-98. ACM Press, 2009. 
24. Jacques Klein, Loic Hélouet, and Jean-Marc Jézéquel. Semantic-based weaving of scenarios. In AOSD, pages 27-38. ACM Press, 2006.

25. Max E. Kramer, Jacques Klein, Jim R. H. Steel, Brice Morin, Jörg Kienzle, Olivier Barais, and Jean-Marc Jézéquel. Achieving practical genericity in model weaving through extensibility. In 6th International Conference on Model Transformation (ICMT'13), volume 7909 of LNCS, pages 108-124. Springer Berlin Heidelberg, 2013.

26. Florent Latombe, Xavier Crégut, Benoît Combemale, Julien Deantoni, and Marc Pantel. Weaving Concurrency in eXecutable Domain-Specific Modeling Languages. In 8th ACM SIGPLAN International Conference on Software Language Engineering (SLE), Pittsburg, United States, 2015. ACM.

27. Edward A Lee and Alberto Sangiovanni-Vincentelli. The tagged signal model-a preliminary version of a denotational framework for comparing models of computation. Memorandum $U C B / E R L M, 96: 71,1996$.

28. Frédéric Mallet, Julien Deantoni, Charles André, and Robert De Simone. The Clock Constraint Specification Language for building timed causality models. Innovations in Systems and Software Engineering, 6(1-2):99-106, March 2010.

29. Jonathan Marchand, Benoit Combemale, and Benoit Baudry. A Categorical Model of Model Merging and Weaving. In 4th International Workshop on Modelling in Software Engineering (MiSE 2012). IEEE, 2012.

30. Ashley McNeile and Nick Simons. Protocol modelling.: A modelling approach that supports reusable behavioural abstractions. SoSyM, 5(1):91-107, 2006.

31. Sébastien Mosser, Mireille Blay-Fornarino, and Robert France. Workflow design using fragment composition. In TAOSD VII, volume 6210, pages 200-233. 2010.

32. Gunter Mussbacher. Aspect-Oriented User Requirements Notation. PhD thesis, University of Ottawa, Canada, 2010.

33. Gunter Mussbacher, Omar Alam, Mohammad Alhaj, Shaukat Ali, Nuno Amálio, Balbir Barn, Rolv Bræk, Tony Clark, Benoit Combemale, Luiz Marcio Cysneiros, Urooj Fatima, Robert France, Geri Georg, Jennifer Horkoff, Jörg Kienzle, Julio Cesar Leite, Timothy C. Lethbridge, Markus Luckey, Ana Moreira, Felix Mutz, A. Padua A. Oliveira, Dorina C. Petriu, Matthias Schöttle, Lucy Troup, and Vera M. B. Werneck. Assessing composition in modeling approaches. In Workshop CMA'12. ACM, 2012.

34. Gunter Mussbacher, Daniel Amyot, and Jon Whittle. Composing Goal and Scenario Models with the Aspect-Oriented User Requirements Notation Based on Syntax and Semantics. In Aspect-Oriented Requirements Engineering. Springer Berlin Heidelberg, 2013.

35. Shiva Nejati, Mehrdad Sabetzadeh, Marsha Chechik, Steve Easterbrook, and Pamela Zave. Matching and merging of statecharts specifications. In ICSE, 2007.

36. Mogens Nielsen, Gordon Plotkin, and Glynn Winskel. Petri nets, event structures and domains. In Semantics of Concurrent Computation, volume 70 of LNCS. Springer Berlin Heidelberg, 1979.

37. Object Management Group. Unified Modeling Language (v2.5.0), March 2015.

38. OMG. Uml infrastructure specification v2.4, 2010.

39. D. L. Parnas. On the criteria to be used in decomposing systems into modules. Communications of the Association of Computing Machinery, 15(12):1053-1058, 1972.

40. Matias Vara Larsen and Arda Goknil. Railroad Crossing Heterogeneous Model. In GEMOC workshop 2013 - International Workshop on The Globalization of Modeling Languages, Miami, Florida, USA, September 2013.

41. Matias Ezequiel Vara Larsen, Julien Deantoni, Benoit Combemale, and Frédéric Mallet. A Behavioral Coordination Operator Language (BCOoL). In Timothy Lethbridge, Jordi Cabot, and Alexander Egyed, editors, International Conference on Model Driven Engineering Languages and Systems (MODELS), number 18, page 462, Ottawa, Canada, September 2015. ACM. to be published in the proceedings of the Models 2015 conference.

42. Jon Whittle, Praveen Jayaraman, Ahmed Elkhodary, Ana Moreira, and João Araújo. MATA: A Unified Approach for Composing UML Aspect Models Based on Graph Transformation. In Transactions on Aspect-Oriented Software Development VI, volume 5560 of $L N C S$, pages 191-237. Springer Berlin Heidelberg, 2009.

43. G Winskel. Event structures. In Advances in Petri Nets 1986, Part II on Petri Nets: Applications and Relationships to Other Models of Concurrency. Springer-Verlag New York, Inc., 1987.

44. Gefei Zhang and Matthias M. Hölzl. HiLA: High-level aspects for UML state machines. In MoDELS Workshops, volume 6002 of $L N C S$, pages 104-118. Springer, 2009. 